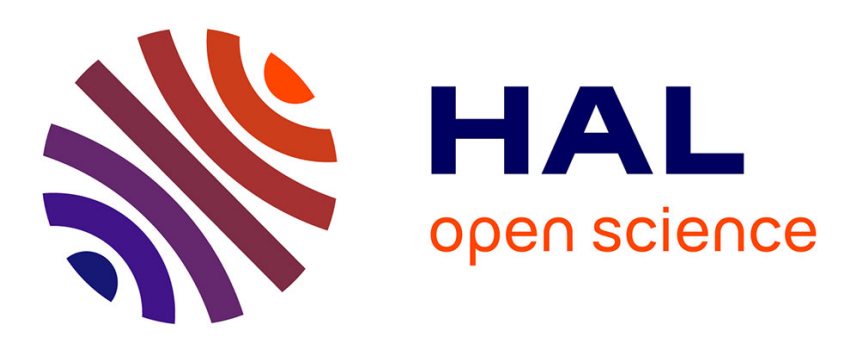

\title{
Design of a nonlinear energy harvester based on high static low dynamic stiffness for low frequency random vibrations
}

Cyril Drezet, Najib Kacem, Noureddine Bouhaddi

\section{- To cite this version:}

Cyril Drezet, Najib Kacem, Noureddine Bouhaddi. Design of a nonlinear energy harvester based on high static low dynamic stiffness for low frequency random vibrations. Sensors and Actuators A: Physical , 2018, 283, pp.54-64. 10.1016/j.sna.2018.09.046 . hal-02130053

\section{HAL Id: hal-02130053 https://hal.science/hal-02130053}

Submitted on 15 May 2019

HAL is a multi-disciplinary open access archive for the deposit and dissemination of scientific research documents, whether they are published or not. The documents may come from teaching and research institutions in France or abroad, or from public or private research centers.
L'archive ouverte pluridisciplinaire HAL, est destinée au dépôt et à la diffusion de documents scientifiques de niveau recherche, publiés ou non, émanant des établissements d'enseignement et de recherche français ou étrangers, des laboratoires publics ou privés. 


\title{
Design of a nonlinear energy harvester based on high static low dynamic stiffness for low frequency random vibrations
}

\author{
C. Drezet ${ }^{\mathrm{a}, \mathrm{b}}$, N. Kacem ${ }^{\mathrm{a}, *}$, N. Bouhaddi ${ }^{\mathrm{a}}$ \\ ${ }^{a}$ Univ. Bourgogne Franche-Comte, FEMTO-ST Institute, CNRS/UFC/ENSMM/UTBM, Department \\ of Applied Mechanics, 25000 Besançon, France \\ ${ }^{b}$ TRAXENS, 13013 Marseille, France
}

\begin{abstract}
Most of the vibration sources, which could be used for energy harvesting, are vertical and very low frequency (e.g. human movement, vehicle transportation, etc...). Under those conditions and while considering the size constraints, usual vibration energy harvesters (VEHs) underperform due to the combined effect of gravity, mechanical damping and the necessity to be tuned to very low frequencies. In order to overcome these limitations, the concept of High Static Low Dynamic (HSLD) stiffness is proposed and validated for VEHs. To do so, a theoretical study is performed to optimize the electromagnetic structure and design a folded-beam suspension of the moving mass allowing a low mechanical damping. This leads to the design of an original VEH, which is experimentally characterized under harmonic and white noise excitations. Compared to the literature, it demonstrated high performances with up to $41.3 \mathrm{~mW} \mathrm{~cm}^{-3} \mathrm{~g}^{-2}$ of normalized harvested power at an operating low frequency of $5.3 \mathrm{~Hz}$.
\end{abstract}

Keywords:

Vibration Energy Harvesting, High Static Low Dynamic Stiffness, Random Vibrations, Electromagnetic

\section{Introduction}

The Internet of Things (IoT) is a term used to describe a cluster of technologies enabling machine to machine (M2M) communication and machine to human interactions through the Internet [1]. One of these technologies is the autonomous wireless sensors which can be scattered in unfriendly environments leading to communication, electronic or mechanical issues. Power supply is one of the main concerns; due to their limited lifespan and/or their inability to withstand extreme temperatures, usual batteries are sometimes unable to satisfy the sensor specifications. A promising solution is to take advantage of the ambient energy surrounding the sensor: energy harvesting. Among the various exploitable sources [2], this study focuses on the mechanical energy of vibrations.

Vibration energy harvesting aims to turn mechanical vibration into usable electrical power. Most of the vibration energy harvesters can be classified according to their trans-

\footnotetext{
${ }^{*}$ Corresponding author: najib.kacem@femto-st.fr
} 
duction technique: piezoelectric [3], electrostatic [5] or electromagnetic [4] harvesters. The latter enables a high harvested power density for macroscale VEHs with a long life-cycle.

Electromagnetic vibration energy harvesters are often based on a mechanical oscillator responding to an excitation by magnifying the relative motion between a coil of conductive material and an arrangement of magnets. The coil is then immersed in a time varying magnetic field creating an electromotive force (emf) according to Lenz law; electrical energy can then be extracted from the coil. One of the first prototype has been presented by Beeby et al. [6] in 2007, it is based on an electromagnetic structure (magnets and back iron) suspended by a cantilever beam and moving relatively to a Copper coil; with a volume of $0.15 \mathrm{~cm}^{3}$, it is able to generate $46 \mu \mathrm{W}$ from a $55 \mathrm{mg}_{\text {peak }}$ vibration source at $53.2 \mathrm{~Hz}$. Later, Zhu et al. [7] used the same concept with a Hallbach array to enhance the electromechanical coupling; their device is larger $\left(1.28 \mathrm{~cm}^{3}\right)$ and scavenges $157 \mu \mathrm{W}$ from an excitation at $55 \mathrm{mg}_{\text {peak }}, 68 \mathrm{~Hz}$. Marin et al. [8] also based their vibration energy harvester on suspended multiple coils and claim a $55 \%$ increase of the harvested power per unit of volume; their prototype $\left(120 \mathrm{~cm}^{3}\right)$ generates $19.3 \mathrm{~mW}$ when submitted to a $400 \mathrm{mg}_{\text {peak }}$ harmonic vibration at $179 \mathrm{~Hz}$. Cepnik et al. [9] proposed a direct computation of the electromechanical coupling to efficiently design a tubular electromagnetic energy harvester, it generates $20.6 \mathrm{~mW}\left(1 \mathrm{~g}_{\text {peak }}, 50 \mathrm{~Hz}\right)$. In 2009, Mann and Sims [10] introduced the concept of magnetic levitation to functionalize a contact-less stiffness in an electromechanical oscillator. This design has limited performances mainly due to dry friction. In order to overcome this issue, a flexible guidance has been proposed by Mahmoudi et al. [11] which enables a significant reduction of the mechanical damping. When considering magnetic suspensions, Hadas et al. presented a series of devices [12, 13, 14] based on the same design consisting in a rotating arm on which the electromagnetic structure is linked, the stiffness is brought by repelling magnets. The performances of these harvesters have been recently evaluated by Hadas et al. [15]; they respectively produce $6.7 \mathrm{~mW}\left(45 \mathrm{~cm}^{3}\right.$, $\left.420 \mathrm{mg}_{\text {peak }}, 34 \mathrm{~Hz}\right)$ [12], $23 \mathrm{~mW}\left(84 \mathrm{~cm}^{3}, 420 \mathrm{mg}_{\text {peak }}, 17 \mathrm{~Hz}\right)$ [13] and $0.9 \mathrm{~mW}\left(111 \mathrm{~cm}^{3}\right.$, $70 \mathrm{mg}_{\text {peak }}, 28 \mathrm{~Hz}$ ) [14]. Finally, we can mention the work done by Zeng and Khaligh [16] who presented an efficient harvester that uses several industrial techniques (ball bearing, permanent magnet motor like electromagnetic structure); the device $\left(200 \mathrm{~cm}^{3}\right)$ is able to produce $498 \mathrm{~mW}$ from a vibration source of $370 \mathrm{mg}_{\text {peak }}$ at $13 \mathrm{~Hz}$.

The characteristics of vibration energy vary from one source to the other by their direction, frequency range and nature (deterministic or stochastic). This study deals with vertical very low frequency $(1-4 \mathrm{~Hz})$ stochastic excitations that can profusely be found in the environment, especially during human movement [17, 18] or train/truck transportation [19, 20, 21. Note that most of the real word vibration sources have a stochastic nature. In addition, several recent works take the randomness of excitation into account [22, 23, 24].

Numerous solutions have been proposed to efficiently scavenge very low frequency vibration. Frequency-up conversion is a solution aiming to convert very low frequency vibration to more convenient high frequency oscillations [25, 26]. The pendulum motion is also proposed for energy harvesters to reach low frequencies. For instance, Jung et al. [27] presented an energy harvester working at $1 \mathrm{~Hz}$. Magnetic suspension of the VEH moving 
mass enabling low local stiffness is an efficient solution acclaimed by the literature. It has been used by Saha et al. [28] to harvest the energy low frequency human movement. Their $12.7 \mathrm{~cm}^{3}$ device generates $14.55 \mu \mathrm{W}$ from a $420 \mathrm{mg}_{\text {peak }}$ vibration source at $8 \mathrm{~Hz}$, although they identified human walking vibration source to be the most energetic at 2.75 Hz. Aiming to harvest the vibrations from a freight train $(3-5 \mathrm{~Hz})$, De Pasquale et al. [29] also used magnetic suspension. The bulky device having a volume of approximately 1800 $\mathrm{cm}^{3}$ operating at $4.44 \mathrm{~Hz}$ produces $100 \mathrm{~mW}$ when mounted on a train with a velocity of $80 \mathrm{~km} / \mathrm{h}$. Other interesting works on the subject are presented by Munaz et al. [30] and Apo and Priya [31] with prototypes both working at $6 \mathrm{~Hz}$.

Among the above presented solutions, those using magnetic suspension to harvest low frequency excitation are more efficient. However, as illustrated by the numerous presented studies, the resonant frequency can hardly be decreased under $5-6 \mathrm{~Hz}$. In fact, when considering the action of gravity on the harvester (vertical excitation) and a limited volume, those solutions are inadequate. Indeed, the gravitational force implies a high static displacement of the moving part of the harvester which requires a cumbersome device; this explains the bulkiness of De Pasquale et al. [29] $4.4 \mathrm{~Hz}$ device. For example, the static displacement would be $15.5 \mathrm{~cm}$ for a prototype with a linear stiffness tuned to $4 \mathrm{~Hz}$ and $62.1 \mathrm{~cm}$ at $2 \mathrm{~Hz}$. This problem has been mentioned by Maier et al. 32] when trying to harvest energy from a container on a train; they choose to deal with the less energetic horizontal vibration.

In this paper, we propose a nonlinear VEH based on the HSLD stiffness concept which is commonly used in vibration isolation [33, 34]. It consists in adding a negative stiffness component in parallel with the positive stiffness of a linear oscillator to lower its resonant frequency while maintaining a small static displacement. First, a generic electromagnetic VEH model is introduced and the linear case is analyzed to provide design rules for optimal energy harvesting under random excitation. Then, based on the proposed model, a comparison is carried out between several solutions to harvest vertical very low frequency stochastic excitations under volume constraints. This comparison shows that HSLD stiffness is the most performing solution. This concept has been implemented on VEH prototypes using bistable buckled beams and magnetic repulsion, respectively. The two prototypes have high performances with an operating frequency around $5.3 \mathrm{~Hz}$ and normalized harvested powers of 16.5 and $41.3 \mathrm{mWcm}^{-3} \mathrm{~g}^{-2}$, respectively.

\section{Theoretical background}

\subsection{Generic model}

In this paper, we propose a generic model of an electromagnetic vibration energy harvester as illustrated in Figure 1. This model is established on a vertical damped single degree of freedom oscillator under base excitation (mass $m$, elastic potential energy $U_{e l}$, damping coefficient $c_{m}$ ). The displacement of the moving mass and the base respctively to the reference frame are respectively called $x$ and $y$ and their difference is $z=x-y$. $g$ is the gravity constant. The electromechanical coupling is done by a coil (fixed to the base) made out of conductive material and placed in the vicinity of the moving mass assimilated to a magnet, the interaction between coil and magnet is then characterized 


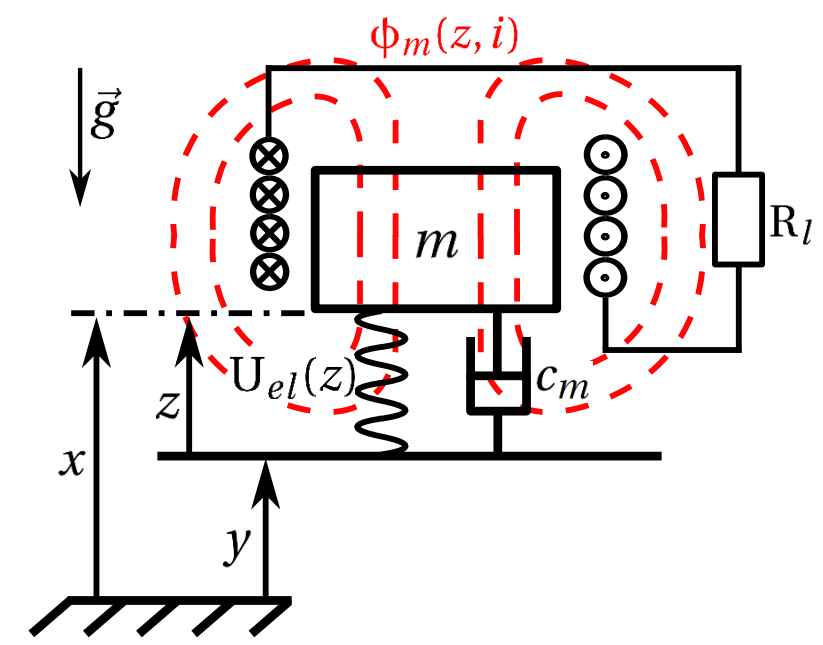

Figure 1: Electromagnetic vibration energy harvester generic model

by the magnetic flux $\phi_{m}$ which depends on the relative displacement $z$ and the current flowing in the coil $i$.

The equation characterizing the displacement $z$ of the mass is given as follows:

$$
m \ddot{z}+c_{m} \dot{z}+\frac{\mathrm{d} U_{e l}(z)}{\mathrm{d} z}-\frac{\partial \phi_{m}}{\partial z} \cdot i=-m g-m \ddot{y}
$$

where the dots describes the derivation with respect to time. The behavior of the vibration energy harvester is governed by the following coupled equations:

$$
\left\{\begin{array}{l}
m \ddot{z}+c_{m} \dot{z}+\frac{\mathrm{d} U_{e l}(z)}{\mathrm{d} z}+\gamma n i=-m g-m \ddot{y} \\
\left(n^{2} R_{0}+R_{l}\right) \cdot i=\gamma n \dot{z}
\end{array}\right.
$$

The parameters of Equation (2) are given in Appendix A.1. From this system, one can obtain the main figure of interest which is the instantaneous harvested power $P$ :

$$
P=R_{l} i^{2}
$$

The advantage of the model presented above is its ability to describe the behavior of a whole range of single degree of freedom electromagnetic vibration energy harvesters. Actually, as shown in Figures $2 \mathrm{a}$ and $2 \mathrm{~b}$, the elastic potential energy $U_{e l}$ may describe linear or nonlinear (hardening, multistable, asymmetric, HSLD, etc.) behavior and it can even include stopper forces to represent the spatial limitations.

\subsection{Linear harvester under random excitation}

To use it as a reference and introduce some basic principles, it is interesting to deal with the case of a linear harvester. The elastic potential energy $U_{e l}$ is then equal to $0.5 k z^{2}$ where $k$ is the linear stiffness coefficient.

Dealing with the linear harvester without displacement constraints, the action of gravity only adds an offset $z_{0}$ to the displacement. Introducing the resonant frequency $\omega_{0}$ of the harvester which is equal to $\sqrt{k / m}$, allows to express $z_{0}$ : 


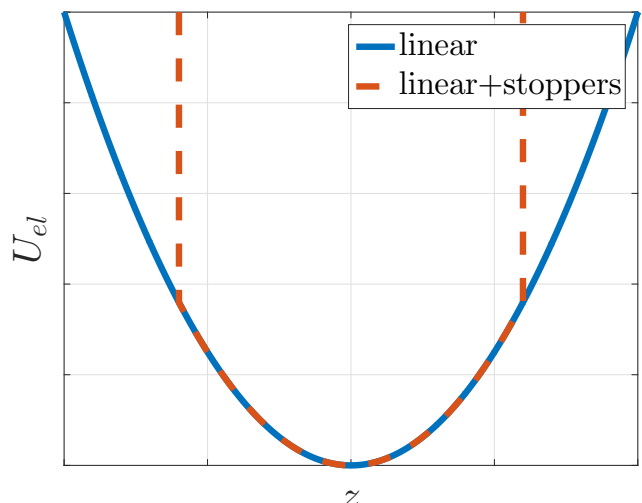

(a) $U_{e l}$ shape for linear harvester

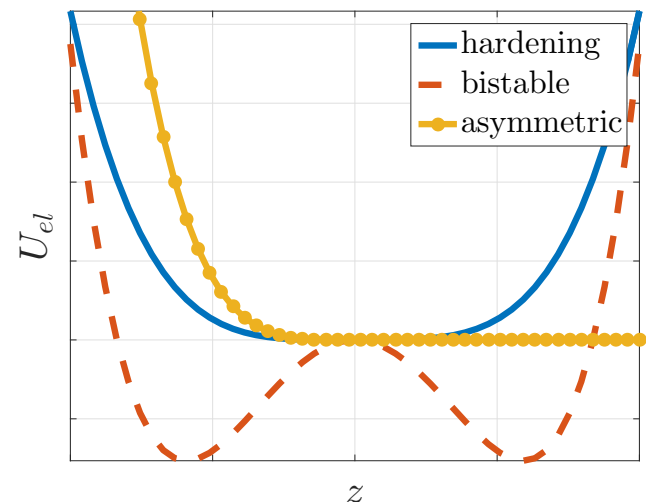

(b) $U_{e l}$ shape for nonlinear harvester

Figure 2: Elastic potential energy for several harvester types

$$
z_{0}=-\frac{g}{\omega_{0}^{2}}
$$

This expression shows that for high eigenfrequencies, the static displacement $z_{0}$ is low and the term $-m g$ can be neglected in equation (2).

$\ddot{y}$ is defined as a stationary Gaussian noise characterized by its single-sided Power Spectral Density (PSD) denoted by $S_{\ddot{y} \ddot{y}}$ which depends on the frequency $\omega$. As we aim to harvest the vibration energy during a relatively long period, the probabilistic steady-state analysis is used. The main details and notations are given in Appendix A.2.

When impedance matching is satisfied, the mean harvested power $\widetilde{P}_{\max }$ can be expressed as follows:

$$
\widetilde{P}_{\max }=\frac{\pi S_{0} m}{2} \cdot \eta_{w n}
$$

Where $\eta_{w n}$ is an efficiency coefficient which tends to 1 when $\gamma^{2} / R_{0} c_{m}$ increases; its expression is given by:

$$
\eta_{w n}=\frac{\gamma^{2} / R_{0} c_{m} \sqrt{1+\gamma^{2} / R_{0} c_{m}}}{\left(1+\sqrt{1+\gamma^{2} / R_{0} c_{m}}\right) \cdot\left(1+\gamma^{2} / R_{0} c_{m}+\sqrt{1+\gamma^{2} / R_{0} c_{m}}\right)}
$$

Equation (5) implies that the maximum power which can be harvested from a linear harvester under white noise vibration equals $\pi S_{0} m / 2$. This result is in accordance with the work done by Langley [23] who also demonstrated that this bound holds for any vibration energy harvester.

In order to increase the harvested power, the moving mass $m$ and $\eta_{w n}$ must be maximized according to equation (5). Equation (6) shows that to maximize $\eta_{w n}$, the intrinsic coupling $\gamma$ must be as high as possible while maintaining small coil intrinsic resistance 
$R_{0}$ and small mechanical damping $c_{m}$. Those general design rules are used in the design of the proposed prototype.

While $\gamma^{2} / R_{0} c_{m}$ is positive, we have $R_{\text {lopt wn }}<R_{\text {lopt harm }}$. This result is important since it reflects the fact that optimizing a linear harvester to exploit random vibration leads to the best compromise between maximum power and bandwidth size. When dealing with large-band random excitation, and more particularly with white noise, the best harvester will be the one having the biggest area under its frequency response in terms of power which implies a compromise between maximum power and bandwidth. $R_{\text {lopt wn }}<$ $R_{\text {lopt harm }}$ means that the global damping is higher in the case of white noise excitation which implies that the bandwidth is larger. Therefore, it would be interesting to use the mean power harvested $\left(\widetilde{P}_{\max }\right)$ under white noise normalized $\left(\right.$ i.e. $\left.\eta_{w n}\right)$ to compare vibration energy harvesters because it takes the bandwidth into account and does not depend on the operating frequency $\omega_{0}$; which is not the case of usual figure of merit [38].

\section{Benefits of HSLD concept}

\subsection{HSLD stiffness}

As expressed by equation (4), the vertical linear energy harvester implies a static displacement due to gravity that increases its volume when tuned to very low frequencies jeopardizing its performances when the displacement is limited $\left(z_{0}\right.$ equals $62.1 \mathrm{~cm}$ at 2 $\mathrm{Hz}$ ). To overcome this issue, we propose to use the technique called HSLD stiffness. It consists in adding a negative stiffness component in parallel with the positive stiffness of a linear oscillator to lower its resonant frequency while maintaining a small static displacement.

Using the generic model, this technique results in the mechanical stiffness defined by:

$$
\frac{\mathrm{d} U_{\text {elhsld }}(z)}{\mathrm{d} z}=k z-k_{\text {neg }}\left(z-z_{0}\right)+k_{3}\left(z-z_{0}\right)^{3}
$$

In this equation, $k$ and $z_{0}$ are characteristics of the linear harvester, $k_{n e g}$ is the negative stiffness component and $k_{3}$ is the stiffening nonlinear term which makes the negative stiffness realistic. Note that to ensure a monostable behavior, we have $k_{n e g}<k$.

Then, the static and dynamic stiffness are dissociated. In fact, static displacement is unchanged (given by equation (4)) and the static stiffness is $k$. While the dynamic stiffness, which is defined by the slope of the stiffness curve around $z_{0}$, is equal to $k-k_{n e g}$. This means that by choosing an initial linear harvester with high stiffness $k$, we obtain a small static displacement $z_{0}$; then the negative stiffness $k_{n e g}$ is set in a way to tune the harvester to the excitation frequency. The resonant frequency of the harvester with HSLD stiffness is given by:

$$
\omega_{0 h s l d}=\sqrt{\frac{k-k_{n e g}}{m}}
$$




\subsection{Comparative study}

To illustrate the benefit brought by HSLD stiffness in vibration energy harvesting, we consider a design case for which the vibration source is assumed to be a vertical stationary gaussian noise. The performances of four types of vibration energy harvesters under the condition presented in Appendix B are compared.

For each type of harvester, the parameters of the elastic potential energy are bounded to ensure that the equilibrium position lies in the displacement range. For each configuration, the load resistance $R_{l}$ maximizing the harvested power is computed using numerical simulations for the design parameters listed in Table1. Doing so, the optimal set of elastic potential energy parameters for each type of harvester are obtained.

Table 1: Values of design parameters used for numerical simulations

\begin{tabular}{ccc}
\hline Parameter & Unit & Value \\
\hline$m$ & $k g$ & $40 \cdot 10^{-3}$ \\
$k$ & $N / m$ & $14 \cdot 10^{3}$ \\
$c_{m}$ & $N . s / m$ & $57 \cdot 10^{-2}$ \\
$\gamma$ & $N / A$ & $28 \cdot 10^{-3}$ \\
$n$ & $t u r n$ & 628 \\
$R_{0}$ & $\mu \Omega$ & 11 \\
\hline
\end{tabular}

The case of the linear harvester without the constrained displacement (no stoppers) is used as a reference, it is optimized when tuned to $\omega_{\max }$. The normalized power extracted from these optimal configurations is given in Figure 3. From these results, the vibration energy harvester with the HSLD stiffness has the best performances.

To comment those results, it is interesting to first discuss the effect of the stoppers on the harvester's response. Impacts of the moving mass on the stoppers, modeled by stiff elastic contacts (equation (B.2) ), imply a strong hardening of the response of the harvester. Thus, the moving mass tends to capture high frequency energy $\left(\omega>>\omega_{\max }\right)$ which is small in our example. Therefore, recurrent impacts with the stoppers decrease the energy harvesting performances.

By minimizing the effect of gravity, the harvester with HSLD stiffness can be tuned to $\omega_{\max }\left(=\omega_{0 h s l d}\right)$ and oscillate with almost no contact with the stoppers (nonlinear stiffness $k_{3}$ must be null for optimal harvesting). Hence, it is able to harvest $80 \%$ of the power produced by the linear harvester without displacement constraints.

Showing good but poorest performances, the linear harvester has to be tuned to 1.2 $\omega_{\max }$ to limit the hardening effect of stoppers despite the $z_{\max }$ offset added to the equilibrium position to reflect design consideration.

The harvester with Duffing nonlinearity has low harvested power. In fact, its best performance is when the nonlinearity is null and the oscillator is tuned to $1.6 \omega_{\max }$. The tuning frequency being too high, the vibration source is not correctly exploited. 


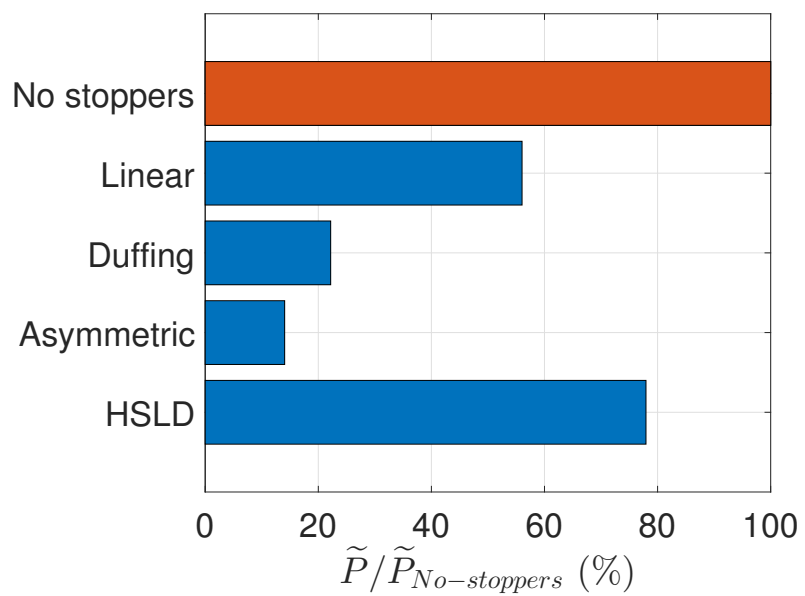

Figure 3: Normalized performances of the different types of harvester

Finally, the harvester with asymmetric stiffness implies a high local stiffness to have an equilibrium position within the $\pm z_{\max }$ range. At its best, it is tuned to $2 \omega_{\max }$ which explains its very poor performances. For the four configurations, the optimal values of tuning parameters are listed in Table 2 .

Table 2: Optimal values of tuning parameters

\begin{tabular}{cc}
\hline No Stoppers & $\omega_{0}=\omega_{\text {max }} / R_{l}=610 \Omega$ \\
Linear & $\omega_{0}=1.2 \omega_{\text {max }} / R_{l}=170 \Omega$ \\
Duffing & $\omega_{0}=1.57 \omega_{\text {max }} / k_{3}=0 N . m^{-3} / R_{l}=610 \Omega$ \\
Asymmetric & $z_{\text {mag }}=-z_{\text {max }-1 m m} / F_{\text {mag }}=62 m N . m^{2} / h=4.5 m m / R_{l}=610 \Omega$ \\
HSLD & $\omega_{0 H S L D}=\omega_{\text {max }} / k_{3}=0 N . m^{-3} / R_{l}=460 \Omega$ \\
\hline
\end{tabular}

This study shows that VEH based on HSLD concept offers high performances compared to other solutions in the following conditions: the vibration source is vertical; the VEH operates at very low frequency and the displacement of the moving mass is constrained.

\section{Proposed design and experimental protocol}

The last part of this paper aims to propose a novel VEH based on HSLD concept and highlight its experimental performances. First, the introduced design rules are used to obtain a high performances linear vibration energy harvester. Then the feasibility of introducing HSLD stiffness to the linear VEH through bistable buckling beams and also magnetic repulsion forces is demonstrated. The obtained results are in good agreement with the theoretical model.

\subsection{Linear harvester}

If we refer to the design rules established in the theoretical part, apart from the maximization of the moving mass $m$ and the tuning of the resonant frequency $\omega_{0}$, it is essential to maximize the term $\gamma^{2} / R_{0} c_{m}$. This term is split in two parts when adopting a two steps 


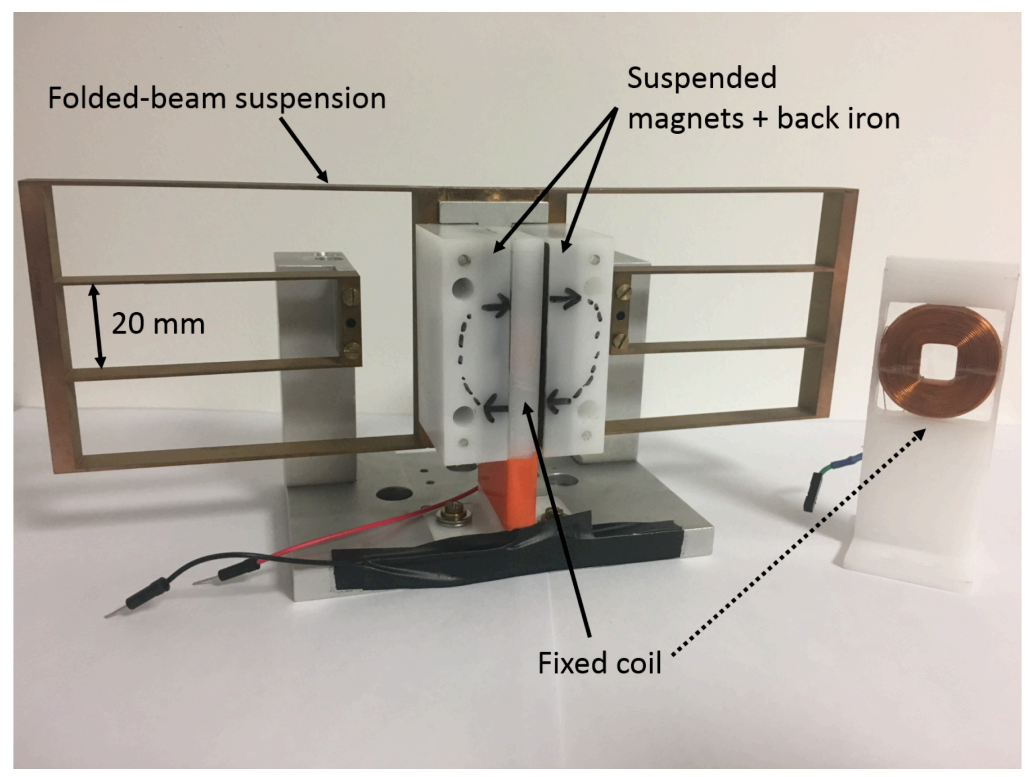

Figure 4: Fabricated linear prototype

design: the electromagnetic structure sets $\gamma^{2} / R_{0}$ and the adequate guidance/suspension of the moving mass allows a low mechanical damping $c_{m}$.

The first step concerns the multiobjective optimization of the electromagnetic structure, which is detailed in Appendix C. The two objective functions being conflictual (i.e. when $f_{1}$ is $\max , f_{2}$ is min and vis-versa), the final results of this optimization problem contain 30 Pareto-optimal solutions given by the Pareto front in Figure C.3. One compromise solution is $\gamma^{2} / R_{0}=16 \mathrm{~N}^{2} . \mathrm{W}^{-1}$ and $\Delta z_{\gamma}=9.1 \mathrm{~mm}$, which is given in Table 3 .

Table 3: Optimal design parameters $(\mathrm{mm})$

\begin{tabular}{lllll}
\hline$H_{\text {aimant }}$ & $H_{\text {air }}$ & $h_{Z}$ & $h_{Y}$ & $e$ \\
\hline 2.5 & 4.6 & 30 & 26 & 10 \\
\hline
\end{tabular}

The second step of the design concerns the guidance/suspension of the moving mass which generates both a pure vertical translation and a smallest mechanical damping $c_{m}$. The use of a folded-beam suspension, very popular in MEMS design [41, 42, is chosen because it offers a pure translation and a linear stiffness, while maintaining a high life cycle.

The linear vibration energy harvester prototype is given in Figure 4, it has an overall volume of $250 \mathrm{~cm}^{3}$. The fabricated suspension is made out of one piece of Beryllium Copper.

Using several characterization experiments including harmonic and white noise excitation tests, the performances of this prototype have been identified. It displays a linear behavior over a large dynamic range with a resonant frequency of $16.6 \mathrm{~Hz}$. The prototype demonstrates high performances with a low mechanical damping of $0.06 \%$ enabling the 


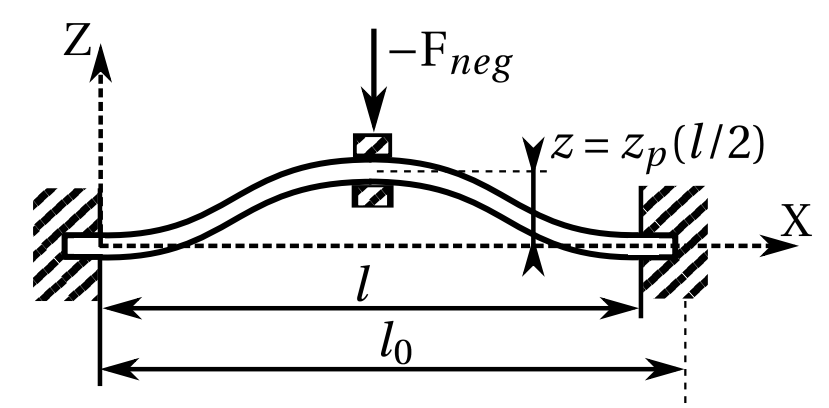

Figure 5: Bistable buckled beam model

extraction of $1.49 \mathrm{~mW}$ mean power with a load resistance $R_{l}=1.1 \mathrm{k} \Omega$ under a $7 \mathrm{mg}_{\text {peak }}$ harmonic excitation. When it is subjected to $4 \times 10^{-5} \mathrm{~g}^{2} \cdot \mathrm{Hz}^{-1}$ white noise excitation, it shows an efficiency coefficient $\eta_{w n}$ of $75 \%$, which corresponds to a harvested power of 323 $\mu \mathrm{W}$ with a load resistance $R_{l}=0.2 k \Omega$.

Notably and as predicted by the model, the optimal value of $R_{l}$ is lower when dealing with white noise compared to a harmonic excitation which implies a larger harvesting bandwidth.

\subsection{Harvester with mechanical HSLD stiffness}

As mentioned in the theoretical part, introducing HSLD stiffness in a vibration energy harvester is an interesting alternative when dealing with very low frequency vertical vibration sources. The most popular method to do so is to use the negative stiffness brought by bistable buckled beams [33]. As shown in Figure 5, a moving mass attached to the center of a doubly-clamped buckled beam applies a force characterized by a negative stiffness.

To enhance the low frequency performances of the proposed linear prototype, we designed and used two parallel buckled beams having a low mechanical stress for a given negative stiffness. The design of those beams aims to obtain a negative stiffness $k_{n e g}$ as close as possible to the linear stiffness of the prototype; i.e. the resonance frequency decreases drastically as described by equation (8).

The stiffness curve of the buckled beam is obtained using an approach similar to the one proposed by Cazottes et al. [43. As displayed in Figure 5, the beam with an initial length $l_{0}$ is compressed to a final length $l$ under a load $-F_{\text {neg }}$ applied at its center which leads to a bending shape $z_{p}$. The stiffness $k_{n e g}$ is identified as the slope of the curve $F_{n e g}$ vs $z_{p}(l / 2)$.

Considering only the buckling modes 1 and $3, z_{p}(x)$ is defined as follows:

$$
z_{p}(x)=a_{1}\left(1-\cos \left(2 \pi \frac{x}{l}\right)\right)+a_{3}\left(1-\cos \left(4 \pi \frac{x}{l}\right)\right)
$$

For a given value of the load $F_{n e g}$, the unknown coefficients $a_{1}$ and $a_{3}$ are identified by the static equilibrium conditions of the beam given by: 


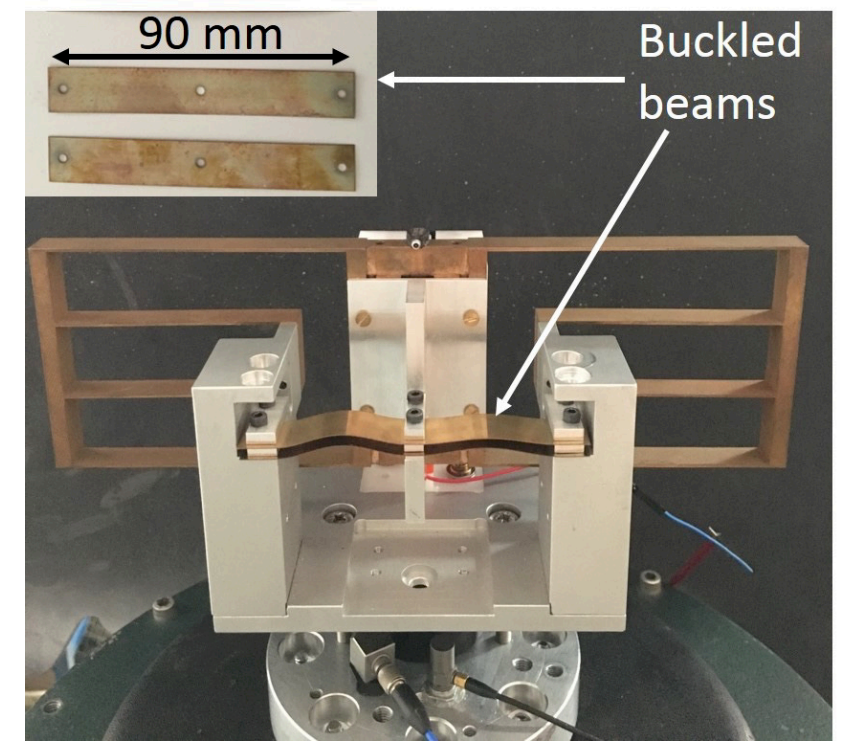

Figure 6: Linear prototype with mechanical HSLD stiffness

$$
\left\{\frac{\partial U_{t o t}}{\partial a_{1}}\left(a_{1}, a_{3}\right)=0, \frac{\partial U_{t o t}}{\partial a_{3}}\left(a_{1}, a_{3}\right)=0\right\}
$$

Where $U_{\text {tot }}$ is the total energy of the beam involving compression, bending and external force energies.

In order to validate the mechanical HSLD concept, four pairs of buckled beams made out of $0.19 \mathrm{~mm}$ thick Beryllium Copper sheets have been fabricated. Three pairs have a width of $13.2 \mathrm{~mm}$ with 2,3 and $4 \%$ compression ratio $\left(l_{0}-l\right) / l_{0}$, and one pair is $15.2 \mathrm{~mm}$ wide and has a $3 \%$ compression ratio. The proposed prototype containing the bistable buckled beams is given in Figure 6, the coil and magnets are behind the folded beam suspension.

Under a white noise excitation of $4 \times 10^{-5} \mathrm{~g}^{2} \cdot \mathrm{Hz}^{-1}$ and with a $50 \Omega$ load resistance, the experimental results of the four harvesters in terms of resonance frequency and PSD of the generated voltage are illustrated in Table 4 and Figure 7, respectively. Remarkably, the last harvester allows the decrease of the resonant frequency from $16.6 \mathrm{~Hz}$ down to $5.3 \mathrm{~Hz}$ with a negligible effect on the equilibrium position. Note that according to theory [43], the negative stiffness does not change with compression rate (hence $f_{0}$ neither); in the presented results, the chaotic change of $f_{0}$ is linked to the difficulty to experimentally master the beams buckling.

Under a harmonic excitation of $3 \mathrm{mg}_{\text {peak }}$, the last harvester enables the extraction of a mean power of $50.2 \mu \mathrm{W}$ on a $340 \Omega$ load resistance. Compared to the linear harvester, the goal of the HSLD stifness is reached by decreasing the resonance frequency, but the buckled beams increases the mechanical damping factor up to $1.40 \%$, which leads to a loss of $26 \%$ in the harvested power $\tilde{P}_{\max }$. This value of damping factor is obtained through 20 measurements of the logarithmic decrement when the moving mass is oscillating freely. In order to overcome this issue, a harvester with magnetic HSLD stifness will be proposed 


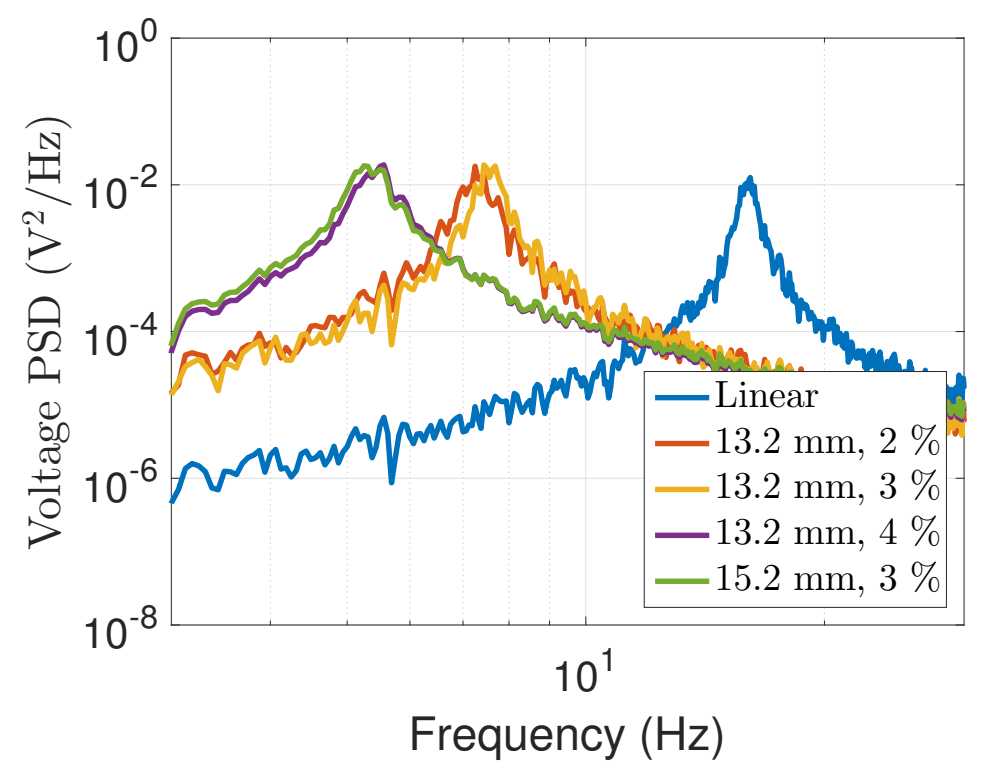

Figure 7: Voltage PSD of the prototype with mechanical HSLD stiffness under white noise

Table 4: Resonant frequency of the different prototypes with mechanical HSLD stiffness

\begin{tabular}{ccc}
\hline $\begin{array}{c}\text { Beams } \\
\text { width }\end{array}$ & $\begin{array}{c}\text { Compression } \\
\text { rate }\end{array}$ & $\begin{array}{c}\text { Measured } \\
f_{0}\end{array}$ \\
\hline mm & $\%$ & $\mathrm{~Hz}$ \\
\hline Linear & $\mathrm{x}$ & 16.6 \\
13.2 & 2 & 7.3 \\
13.2 & 3 & 7.6 \\
13.2 & 4 & 5.5 \\
15.2 & 3 & 5.3 \\
\hline
\end{tabular}

in the next section.

\subsection{Harvester with magnetic HSLD stiffness}

To overcome the disadvantages introduced by the previous design, we proposed the use of the magnetic repulsion phenomenon to introduce the negative term in the HSLD stiffness in the linear VEH shown in Figure 4. The proposed design is given in Figure 8; it consists of 8 pairs of Neodymium repelling each other. It allows high repulsion force on a sufficiently large amplitude range; the tuning variable of the design is the gap $g$ between the chosen magnets.

The proposed prototype, given in Figure 9, is submitted to white noise excitation $\left(4 \times 10^{-5} \mathrm{~g}^{2} \cdot \mathrm{Hz}^{-1}\right)$. The PSD of the voltage measured on a $50 \Omega$ load resistance is given in Figure 10 for several values of the gap $g$. It is shown that a significant decrease of the resonance frequency is reached, thanks to the magnetic HSLD stiffness. In fact, the smaller the gap $g$ is, the stronger is the negative stiffness and the lower is the resonance frequency $f_{0}$. This is depicted in Figure 11 by the relationship between $f_{0}$ and $g$. The smallest 


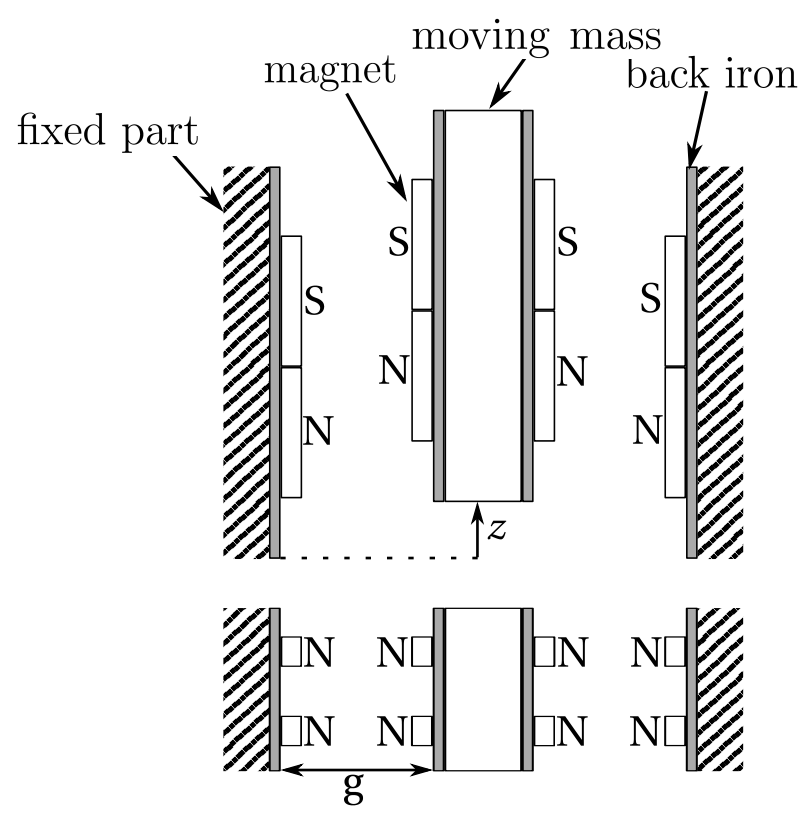

Figure 8: Magnetic negative stiffness design

measured resonance frequency is $5.3 \mathrm{~Hz}$, for $g=13.6 \mathrm{~mm}$. By extrapoling the measured points, we expect to reach much lower frequencies $\left(e . g . f_{0}=2 \mathrm{~Hz}\right.$ at $\left.g=11 \mathrm{~mm}\right)$. The resonance frequency can therefore be tuned by varying the gap between the magnets which is a significant advantage of the magnetic HSLD stiffness.

Under a harmonic excitation of $3 \mathrm{mg}_{\text {peak }}$, the harvester with a resonance frequency $f_{0}=5.3 \mathrm{~Hz}$, a mechanical damping factor of $0.78 \%$ and an efficiency coefficient $\eta_{w n}=59 \%$ enables the extraction of a mean power of $130 \mu \mathrm{W}$ on a $340 \Omega$ load resistance.

\section{Conclusion}

An original high performances vibration energy harvester has been designed, fabricated and experimentally characterized under harmonic and white noise excitations. This VEH is dedicated to vertical vibrations at very low frequencies.

A generic model for a class of electromagnetic vibration energy harvesters has been introduced and used to derive design rules to enhance the performances of the VEH. This design includes a multiobjective optimization of the electromagnetic structure and the use of a folded-beam suspension of the moving mass allowing a low mechanical damping. For very low frequency vibration sources and to minimize the effect of gravity, we implemented an adequate suspension by exploiting the High Static Low Dynamic (HSLD) stiffness concept. The model permitted to highlight the interest of this concept and an experimental protocol has been proposed to validate the design rules on two prototypes using mechanical and magnetic HSLD stiffness respectively. These prototypes have been tested and their performances are summarized in Table 5 and compared to the literature in Figure 12. They display high performances and stands between the best vibration energy harvesters. Nevertheless, the prototype using magnetic HSLD stiffness is more promising than the one using buckled beams HSLD stiffness since it has a better tunability and it 


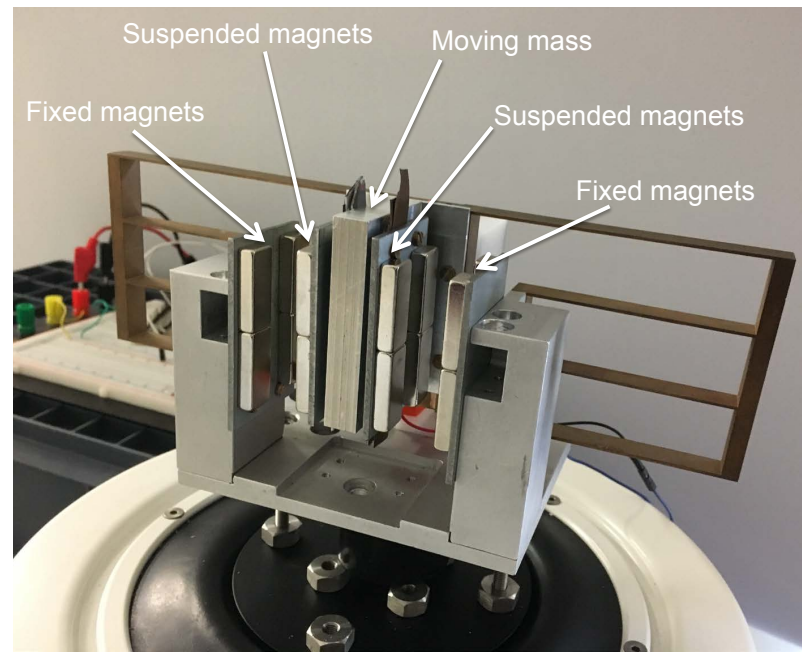

Figure 9: Linear prototype with magnetic HSLD stiffness

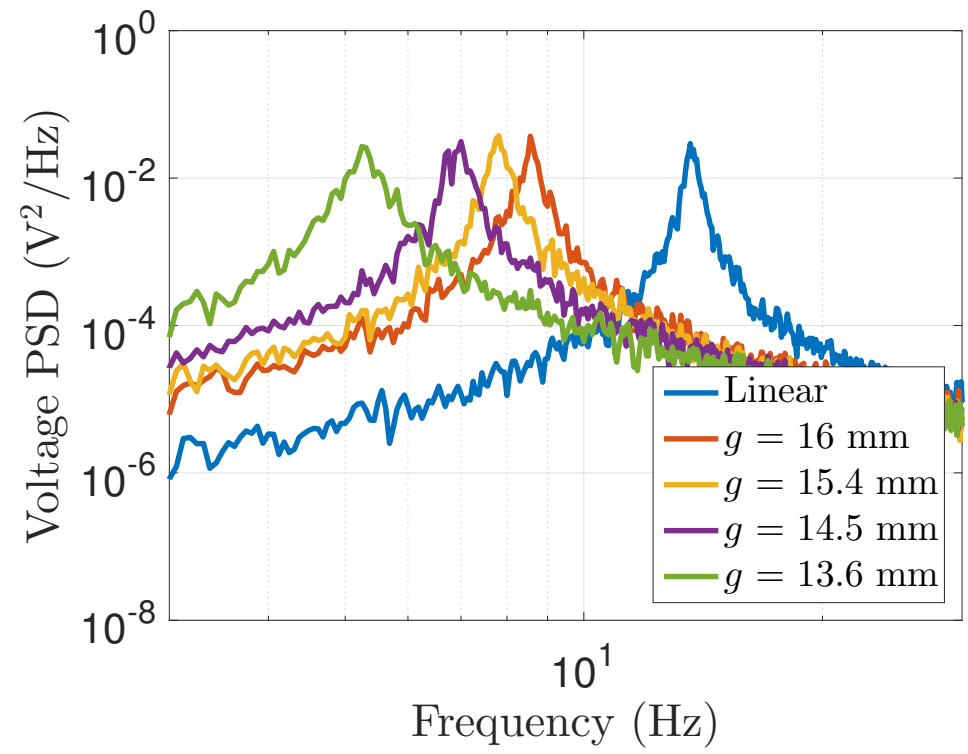

Figure 10: Voltage PSD of the prototype with magnetic HSLD stiffness under white noise 


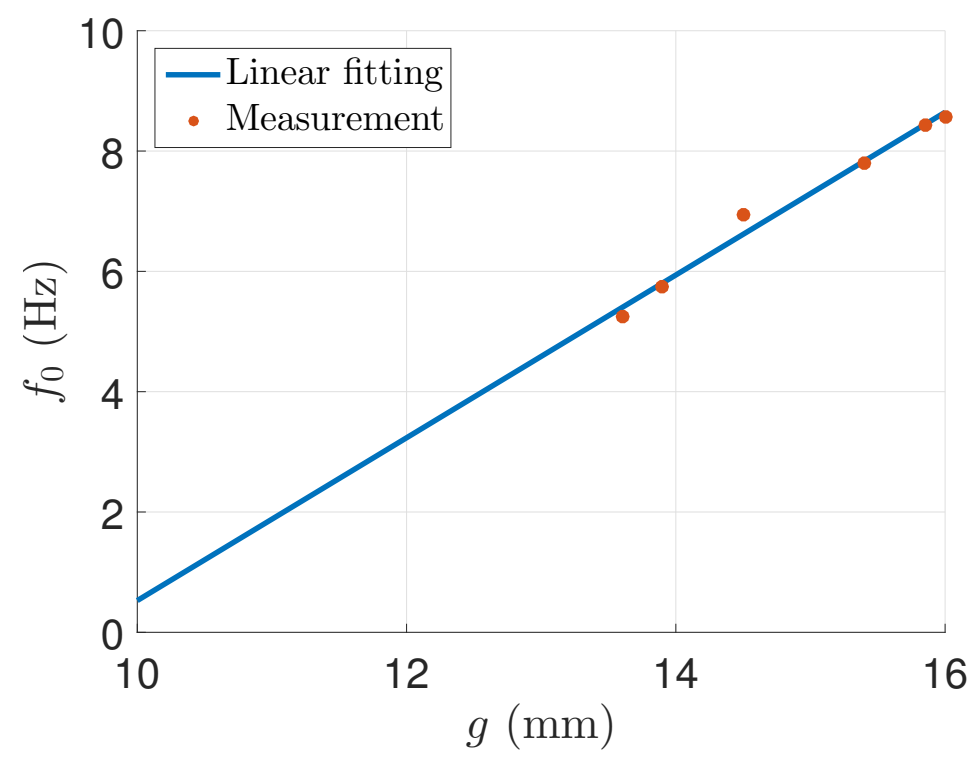

Figure 11: Resonant frequency $f_{0}$ versus $g$ gap

Table 5: Performances of the proposed harvesters under harmonic excitation

\begin{tabular}{lccc}
\hline Prototype & $f_{0}$ & Acceleration & Normalized power \\
& & & \\
\hline Mechanical HSLD & 5.3 & $m g_{\text {peak }}$ & $m W \mathrm{~cm}^{-3} g^{-2}$ \\
Magnetic HSLD & 5.3 & 3 & 16.5 \\
\hline
\end{tabular}

offers high performances with up to $41.3 \mathrm{~mW} \mathrm{~cm}^{-3} \mathrm{~g}^{-2}$ of normalized harvested power at an operating low frequency of $5.3 \mathrm{~Hz}$. 


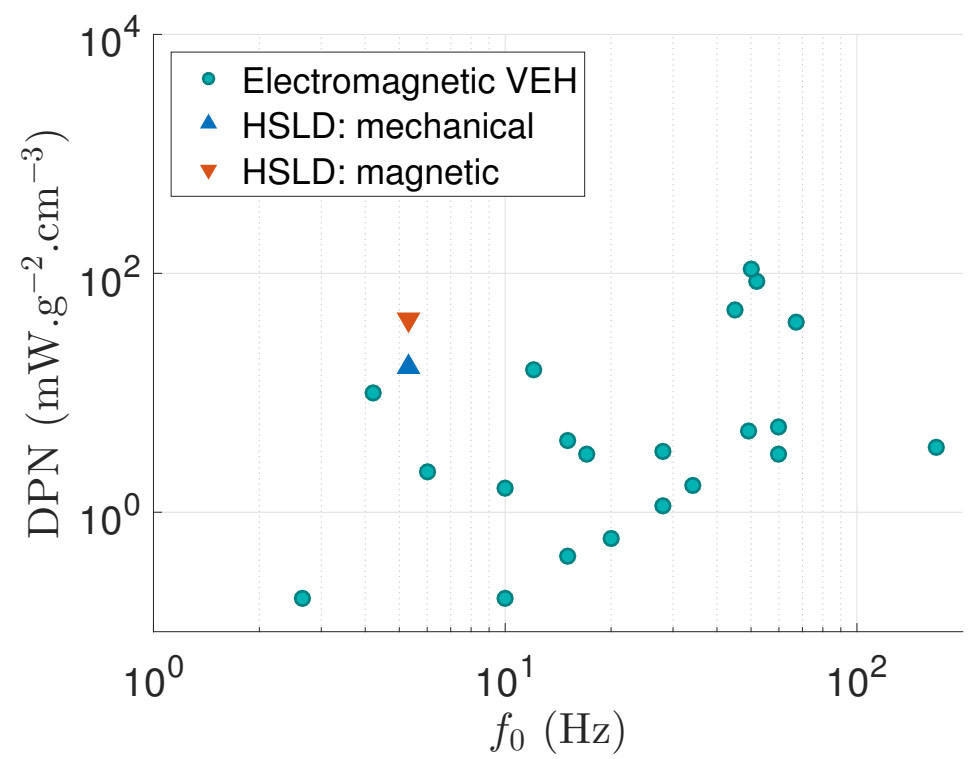

Figure 12: Comparison of the Normalized Power Density of the proposed prototypes with literature [38; the most performant are [29] at $4.2 \mathrm{~Hz}, 30$ at $6 \mathrm{~Hz}, 31$ at $12 \mathrm{~Hz}$ and [6] at $53.2 \mathrm{~Hz}$

\section{Acknowledgements}

This research is supported by the Agence Nationale Recherche Technologie (ANRT) under the CIFRE fund number 2013/1332. This project has been performed in cooperation with the EIPHI Graduate School (contract "ANR-17-EURE-0002"). The authors would also like to thank TRAXENS. 


\section{References}

[1] C. DeFeo, Energy Harvesting and the Internet of Things, Green Information Technology, 2015, pp. 151-160.

[2] F. Shaikh, S. Zeadally, Energy harvesting in wireless sensor networks: A comprehensive review, Renewable and Sustainable Energy Reviews 55 (2016) 1041-1054.

[3] A. Erturk, D. Inman, Piezoelectric Energy Harvesting, John Wiley \& Sons, 2011.

[4] D. Spreeman, Y. Manoli, Electromagnetic Vibration Energy Harvesting Devices, Springer Netherlands, 2012.

[5] S. Boisseau, G. Despesse, B. Ahmed Seddik, Electrostatic Conversion for Vibration Energy Harvesting, in: M. Lallart, Small-Scale Energy Harvesting, Intech, 2012.

[6] S. P. Beeby, R. Torah, M. Tudor, P. Glynne-Jones, T. O’Donnell, C. Saha, S. Roy, A micro electromagnetic generator for vibration energy harvesting, J. of Micromechanics and Microengineering 17 (2007) 1257-1265.

[7] D. Zhu, S. P. Beeby, J. Tudor, N. Harris, Electromagnetic vibration energy harvesting using an improved Hallbach array, The 12th International Workshop on Micro and Nanotechnology for Power Generation and Energy Conversion Applications (2012).

[8] A. Marin, S. Bressers, S. Priya, Multiple cell configuration electromagnetic vibration energy harvester, J. of Physics D: Applied Physics 44 (2011) 416-421.

[9] C. Cepnik, O. Radler, S. Rosenbaum, T. Strhla, U. Wallrabe, Effective optimization of electromagnetic energy harvesters through direct computation of the electromagnetic coupling, Sensors and Actuators A: Physical 167 (2011) 416-421.

[10] B. P. Mann, N. D. Sims, Energy harvesting from the nonlinear oscillations of magnetic levitation, J. of Sound and Vibration 319 (2009) 515-530.

[11] S. Mahmoudi, N. Kacem, N. Bouhaddi, Enhancement of the performance of a hybrid nonlinear vibration energy harvester based on piezoelectric and electromagnetic transductions, Smart Materials and Structures 23 (2014).

[12] Z. Hadas, C. Ondrusek, V. Singule, Electromagnetic vibration power generator, IEEE International Symposium on Diagnostics for Electric Machines, Power Electronics and Drives (2007).

[13] Z. Hadas, J. Kurfurst, C. Ondrusek, V. Singule, Artificial intelligence based optimization for vibration energy harvesting applications, Microsystem Technologies 18 (2012) 1003-1014.

[14] O. Rubes, J. Smilek, Z. Hadas, Development of vibration energy harvester fabricated by rapid prototyping technology, Proc. of the 16th International Conference on Mechatronics Mechatronika (2014) 178-182. 
[15] Z. Hadas, V. Vetiska, J. Vetiska, J. Krejsa, Analysis and efficiency measurement of electromagnetic vibration energy harvester, Microsystem Technologies 22 (2016).

[16] P. Zeng, A. Khaligh, A permanent-magnet linear motion driven kinetic energy harvester, IEEE Transactions on Industrial Electronics 60 (2013).

[17] J. Hamill, T. R. Derrick, K. G. Holt, Shock attenuation and stride frequency during running, Human Movement Science 14 (1995) 45-60.

[18] R. Takeda, S. Tadano, M. Todoh, S. Yoshinari, Human gait analysis using wearable sensors of acceleration and angular velocity, Proc. of the 13th International Conference on Biomedical Engineering (2009).

[19] R. Spinola Barbosa, Vehicle Vibration Response Subjected to Longwave Measured Pavement Irregularity, J. of Mechanical Engineering and Automation 2 (2012) 17-24.

[20] J. Singh, S. P. Singh, E. Joneson, Measurement and analysis of US truck vibration for leaf spring and air ride suspensions, and development of tests to simulate these conditions, Packaging Technology and Science 19 (2006) 309-323.

[21] V. Rouillard, R. Richmond, A novel approach to analysing and simulating railcar shock and vibrations, Packaging Technology and Science 20 (2007) 17-26.

[22] P. L. Green, K. Worden, N. D. Sims, On the identification and modelling of friction in a randomly excited energy harvester, J. of Sound and Vibration 332 (2015) 46964708.

[23] R. S. Langley, Bounds on the vibrational energy that can be harvested from random base motion, J. of Sound and Vibration 339 (2015) 247-261.

[24] Q. He, M. F. Daqaq,Electric load optimization of a nonlinear mono-stable duffing harvester excited by white noise, Meccanica 51 (2016) 1027-1039.

[25] A. Rahimi, O. Zorlu, A. Muhtaroglu, H. Klah, An electromagnetic energy harvesting system for low frequency applications with a passive interface ASIC in standard CMOS, Sensors and Actuators A: Physical 188 (2012) 156-166.

[26] M. Halim, J. Park, Theoretical modeling and analysis of mechanical impact driven and frequency up-converted piezoelectric energy harvester for low-frequency and wide-bandwidth operation, Sensors and Actuators A: Physical 208 (2014) 56-65.

[27] H. Jung, I. Kim, D. Yi Min, A hybrid electromagnetic energy harvesting device for low frequency vibration, Proc. SPIE 8688 (2013).

[28] C. Saha, T. O'Donnell, N. Wang, P. McCloskey, Electromagnetic generator for harvesting energy from human motion, Sensors and Actuators A: Physical 147 (2008) 248-253.

[29] G. De Pasquale, A. Soma, N. Zampieri, Design, simulation and testing of energy harvesters with magnetic suspension for the generation of electricity from freight train vibrations, J. of Computational and Nonlinear Dynamics 7 (2012). 
[30] A. Munaz, B. Lee, G. Chung, A study of an electromagnetic energy harvester using multi-pole magnet, Sensors and Actuators A: Physical 201 (2013) 134-140.

[31] D. Apo, S. Priya, High power density levitation-induced vibration energy harvester, Energy Harvesting and Systems 1 (2014) 1-2.

[32] F. Maier, M. Sturmlechner, S. Dierneder, Novel energy harvester with low friction losses, 6th International Conference on Systems, Signals and Devices (2009) 1-6.

[33] D. L. Platus, Negative-stiffness-mechanism vibration isolation systems, SPIE Vibration Control in Micrelectronics Optics and Metrology 1619 (1991) 44-54.

[34] A. Carrella, M. J. Brennan, T. P. Waters, K. Shin, On the design of a high-staticlow-dynamic stiffness isolator using mechanical springs and magnets, J. of Sound and Vibration 315 (2008) 712-720.

[35] S. Priya, D. J. Inman, Energy Harvesting Technologies, Springer, 2008.

[36] A. Preumont, Random Vibration and Spectral Analysis, Springer, 1994.

[37] N. Stephen, On energy harvesting from ambient vibration, J. of Sound and Vibration 293 (2006) 409-425.

[38] S. Moss, O. Payne, G. Hart, C. Ung, Scaling and power density metrics of electromagnetic vibration energy harvesting devices, Smart Materials and Structures 24 (2015).

[39] C. Drezet, J. Rios Quesada, Energy harvester using a magnetic non-linear spring, Proc. ACTUATOR 14 Bremen (2014) 181-184.

[40] K. Deb, A. Pratap, S. Agarwal and T. Meyarivan, A fast and elitist multiobjective genetic algorithm: NSGA-II, Evolutionary Computation, IEEE Transactions on 6 (2002) 182-197.

[41] S. Shmulevich, I. Hotzen, D. Elata, Dynamically-balanced folded-beam suspensions, 28th IEEE International Conference on MEMS (2015) 215-218.

[42] G. Zhou, P. Dowd, Tilted folded-beam suspension for extending the stable travel range of comb-drive actuators, J. of Micromechanics and Microengineering 13 (2015).

[43] P. Cazottes, A. Fernandes, J. Pouget, M. Hafez, Bistable buckled beam: Modeling of actuating force and experimental validations, J. of Mechanical Design 131 (2009).

\section{Appendix A.}

Appendix A.1.

According to Faraday's law of induction, a voltage called electromotive force $\epsilon$ appears at the coil when the magnetic flux changes $\left(\varepsilon=-d \phi_{m}(z, i) / d t\right)$, it is expressed by the equation: 


$$
\varepsilon=-\left(\frac{\partial \phi_{m}}{\partial z} \frac{\mathrm{d} z}{\mathrm{~d} t}+\frac{\partial \phi_{m}}{\partial i} \frac{\mathrm{d} i}{\mathrm{~d} t}\right)
$$

In equation A.1), the term $\partial \phi_{m} / \partial i$ is often considered as a constant $L$ and called inductance. The coil has its own internal resistance $R_{\text {int }}$ and it is loaded with a resistance $R_{l}$. Then, through Kirchhoff's voltage law, the equation describing the electrical behavior of the device is:

$$
-\left(\frac{\partial \phi_{m}}{\partial z} \frac{\mathrm{d} z}{\mathrm{~d} t}+L \frac{\mathrm{d} i}{\mathrm{~d} t}\right)-R_{\text {int }} \cdot i-R_{l} \cdot i=0
$$

The theoretical analysis of the model involves two main usual approximations. First, as its influence when working in the low frequency domain is small, the inductance $L$ is neglected. Then, the coupling function $-\partial \phi_{m} / \partial z$ is considered constant and proportional to the number of turns $n$ in the coil; it is therefore equal to $\gamma n$ where $\gamma$ is called intrinsic coupling (refered as "Bl" in simple cases). Secondly, for a coil with a fixed volume, the internal resistance $R_{\text {int }}$ is proportional to $n^{2}$ [35]; hence, we have $R_{\text {int }}=n^{2} R_{0}$ with $R_{0}$ the intrinsic resistance of the coil.

Appendix A.2.

The PSD of the displacement $S_{z z}$ can then be expressed as a function of $S_{\ddot{y} \ddot{y}}$ through the mechanical transfer function of the $\mathrm{VEH}$ denoted by $H_{V E H}$ :

$$
S_{z z}(\omega)=\left|H_{V E H}(j \omega)\right|^{2} S_{\ddot{y} \ddot{y}}(\omega)
$$

where

$$
H_{V E H}(j \omega)=\frac{-1}{\omega^{2}-\omega_{0}^{2}+2 j \xi \omega_{0}}
$$

The global damping rate $\xi$ is the sum of mechanical and electrical damping rates which is given by:

$$
\xi=\frac{c_{m}}{2 \sqrt{k m}}+\frac{\frac{(\gamma n)^{2}}{n^{2} R_{0}+R_{l}}}{2 \sqrt{k m}}
$$

The mean harvested power $\widetilde{P}$ can be expressed as:

$$
\widetilde{P}=R_{l} \cdot\left(\frac{\gamma n}{\left(n^{2} R_{0}+R_{l}\right)} \dot{z}_{R M S}\right)^{2}
$$

The mean harvested power $\widetilde{P}$ can then be computed for any stationary Gaussian noise excitation. To obtain reference analytical expression, we use the white noise approximation which holds when the harvester has low damping and is tuned to the most energetic frequency of the noise. The excitation PSD is then constant such as $S_{\ddot{y} \ddot{y}}(\omega)=S_{\ddot{y} \ddot{y m a x}}=S_{0}$ and $\dot{z}_{R M S}$ is expressed by [36]: 


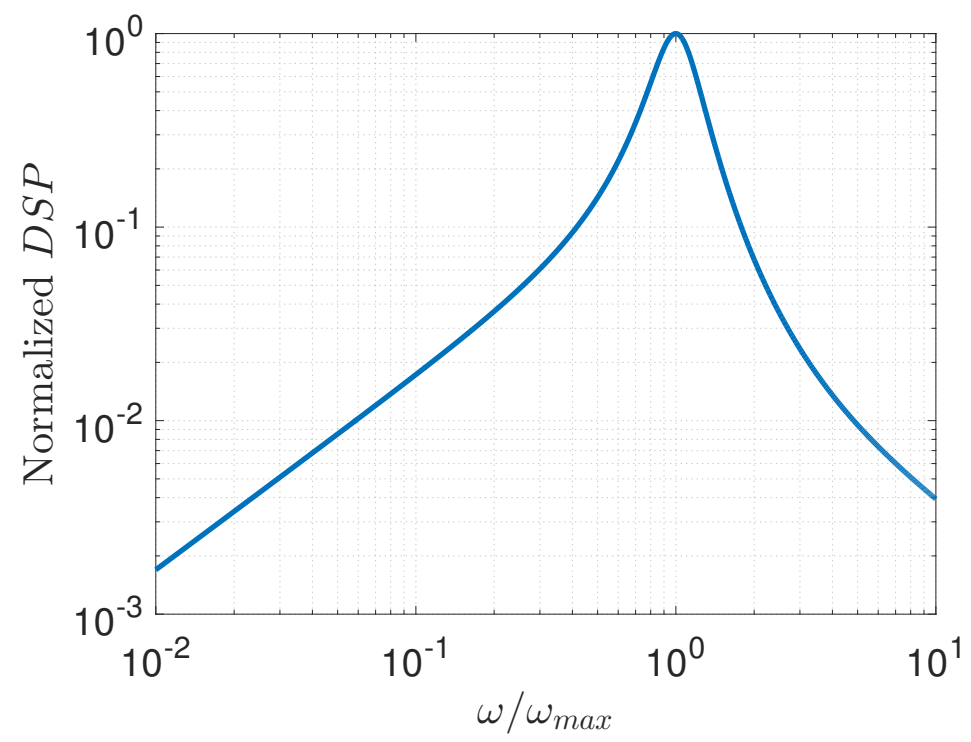

Figure B.1: Normalized Power Spectral Density of the vibration source

$$
\dot{z}_{R M S}=\frac{\pi S_{0}}{4 \xi \omega_{0}}
$$

Combining equations (A.6) and A.7) allows us to obtain the expression of the mean harvested power. This expression is maximized for a specific value of the resistive load $R_{l}$ : this is called impedance matching; the expression of this optimal value in our case is derived:

$$
R_{\text {lopt wn }}=n^{2} R_{0} \sqrt{1+\frac{\gamma^{2}}{R_{0} c_{m}}}
$$

According to Stephen [37], impedance matching under harmonic excitation is given by:

$$
R_{\text {lopt harm }}=n^{2} R_{0}\left(1+\frac{\gamma^{2}}{R_{0} c_{m}}\right)
$$

\section{Appendix B.}

The excitation PSD given in Figure B.1 has a maximum $S_{\max }$ at the frequency $\omega_{\max }$. The displacement of the moving mass is constrained between $\pm z_{\max }$ which is defined by equation (B.1). This is modeled by adding the elastic potential energy of stoppers (equation (B.2) to the actual elastic potential energy of the harvester. The stiffness coefficient of the stoppers $k_{\text {stopper }}$ is chosen to be high enough to constraint the oscillations of the mass.

$$
z_{\max }=\frac{1}{2} \times \frac{g}{\omega_{\max }^{2}}
$$




$$
\frac{\mathrm{d} U_{\text {elstopper }}(z)}{\mathrm{d} z}= \begin{cases}k_{\text {stopper }}\left(z+z_{\max }\right) & \text { if } z \leq-z_{\max } \\ 0 & \text { if }-z_{\max }<z<z_{\max } \\ k_{\text {stopper }}\left(z-z_{\max }\right) & \text { if } z \geq z_{\max }\end{cases}
$$

Apart from the load resistance $R_{l}$ and the elastic potential energy $U_{e l}$, the value of the parameters of the model (equation (2)) are set according to a prototype presented by Drezet and Rios Quesada [39].

The four types of VEHs are differentiated by their elastic potential energy: linear, Duffing with hardening nonlinearity, with asymmetric stiffness and with HSLD stiffness. An example of embodiment for each type is given in Figure B.2.

The elastic potential energy of the linear harvester is given by:

$$
\frac{\mathrm{d} U_{\text {ellin }}(z)}{\mathrm{d} z}=k\left(z-z_{\max }\right)
$$

Note that to avoid the equilibrium position to be out of range, we consider that the mass is suspended by the bottom, which implies that the spring is at rest at $z=z_{\max }$.

For the harvester with hardening Duffing nonlinearity, the elastic potential energy is defined by:

$$
\frac{\mathrm{d} U_{\text {elduff }}(z)}{\mathrm{d} z}=k z+k_{3} z^{3}
$$

Asymmetric stiffness is based on suspending the mass only by a magnetic repulsion at the bottom. The elastic potential energy can then be defined by:

$$
\frac{\mathrm{d} U_{\text {elasym }}(z)}{\mathrm{d} z}=-F\left(\frac{1}{\left(z-z_{\text {mag }}\right)^{2}}+\frac{1}{\left(z-z_{\text {mag }}+2 h\right)^{2}}-\frac{2}{\left(z-z_{\text {mag }}+h\right)^{2}}\right)
$$

where $F$ is a design parameter; $h$ and $z_{m a g}$ are defined in Figure B.2c.

The elastic potential energy of the harvester with HSLD stiffness is given by:

$$
\frac{\mathrm{d} U_{\text {elhsld }}(z)}{\mathrm{d} z}=k z-k_{\text {neg }}\left(z-z_{0}\right)+k_{3}\left(z-z_{0}\right)^{3}
$$

\section{Appendix C.}

The chosen electromagnetic structure is called "Magnet across coil with back iron" (Figure C.1) and identified by Spreeman [4] as one of the most fitted for vibration energy harvesting. It consists in moving a coil of conducting material between two pair of magnets disposed in a way to attract each other; adding back iron on the magnets strengthen the coupling and prevents electromagnetic flux leakage.

To design the electromagnetic structure, we adopt the notations presented in Figure C.2, the chosen coil is square shaped. 


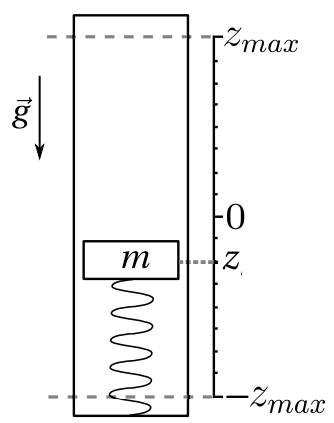

(a) Linear

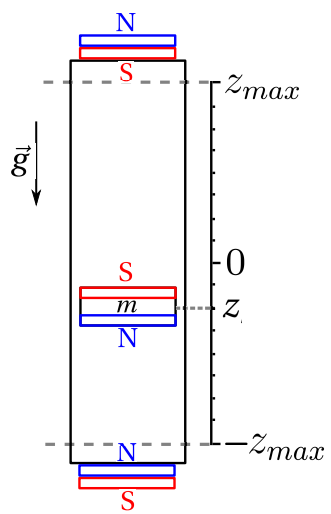

(b) Duffing with hardening nonlinearity

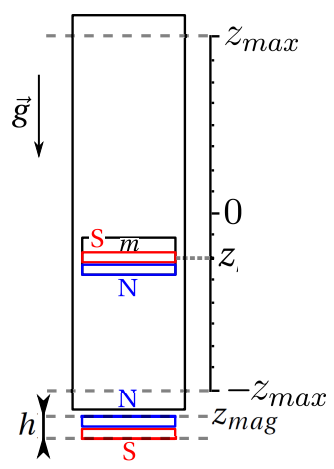

(c) asymmetric

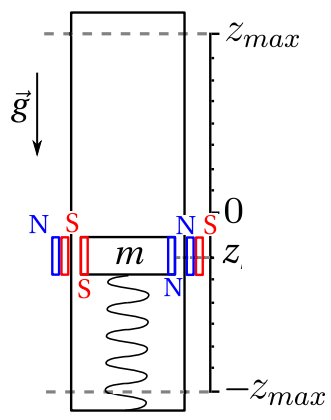

(d) With HSLD stiffness

Figure B.2: Example of embodiement for the four types of harvester 


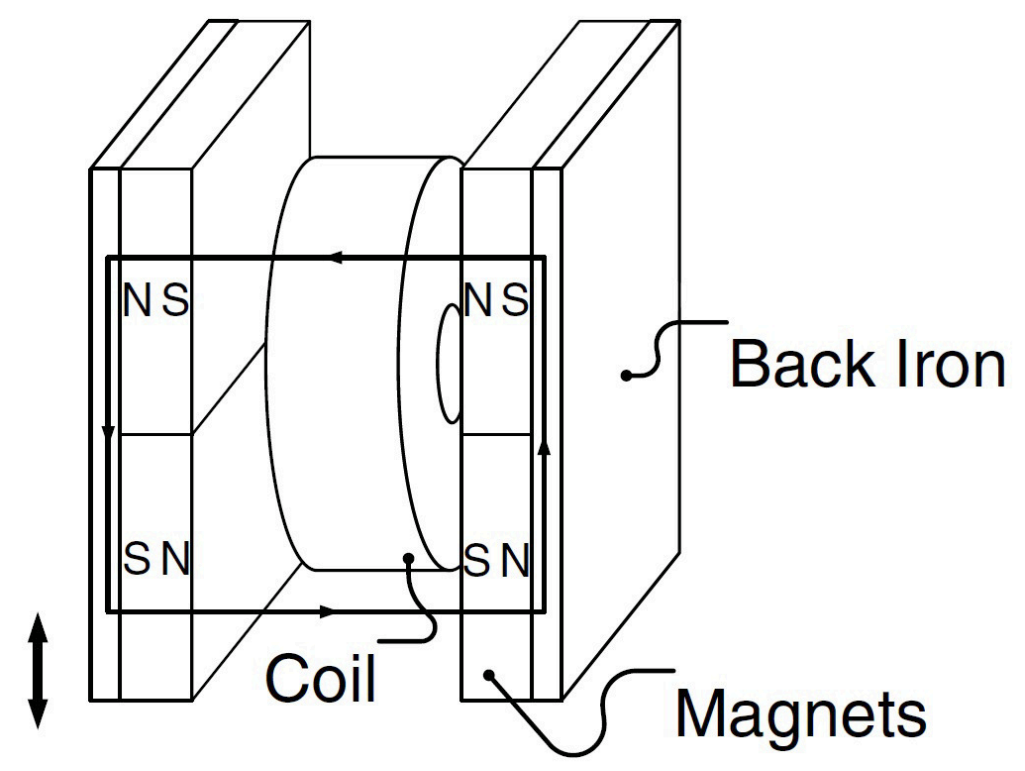

Figure C.1: "Magnet across coil with back iron" structure [4]

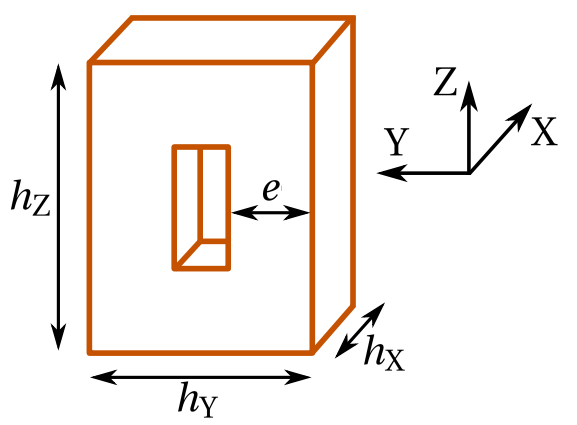

(a) Coil

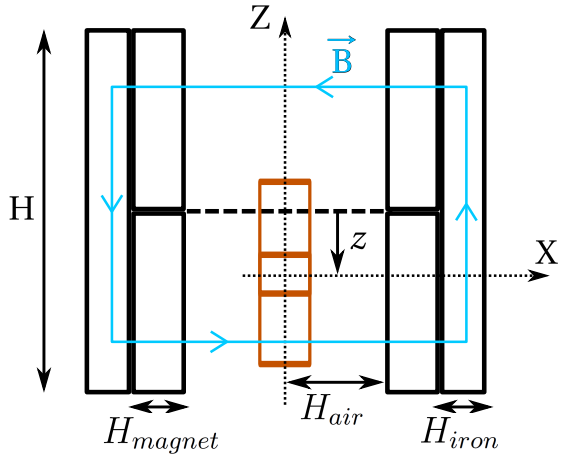

(b) Electromagnetic structure

Figure C.2: Model of the electromagnetic structure 
Using these notations, one can extract the expression of the intrinsic resistance of the coil as follows:

$$
R_{0}=\frac{\rho}{\sigma} \frac{2\left(h_{Y}+h_{Z}-2 e\right)}{e h_{X}}
$$

Where $\rho$ is the resistivity of the coil's material and $\sigma$ is the filling factor of the coil.

Also, by integrating along the $\mathrm{Z}$ axis the component $B_{X}$ of the magnetic field vector $\vec{B}$, one can obtain the expression of the intrinsic coupling as a function of the displacement $z$ as follows:

$$
\gamma(z)=H_{Y} \frac{d}{d z}\left(\int_{-\frac{H_{Z}}{2}+z}^{\frac{H_{Z}}{2}+z} B_{X}\left(h, H, H_{\text {magnet }}, H_{\text {air }}, H_{\text {iron }}\right) d h\right)
$$

Where $B_{X}$ function is obtained through magnetostatic finite element simulations (FEMM software) and $\gamma$ is assumed to be constant over the largest $z$ range.

In order to design the electromagnetic structure, a multiobjective optimization is required: the first objective function to be maximized is $f_{1}=\gamma_{(z=0)}^{2} / R_{0}$; the second objective function to be maximized is $f_{2}=\Delta z_{\gamma}$; The latter is defined as half the range of $z$ on which the intrinsic coupling is over $80 \%$ of its maximal value.

The commonly used materials are Copper for coils and Neodymium for magnets. Consequently, the only variables of the problems are the geometrical dimensions of the

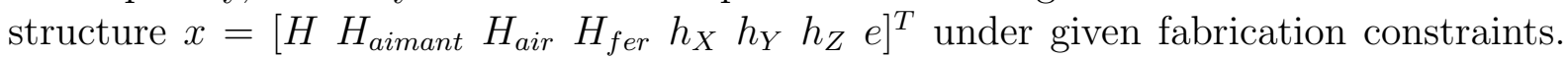
Assuming $H=50 \mathrm{~mm}, h_{X}=2\left(H_{\text {air }}-1 \mathrm{~mm}\right)$ and $H_{\text {fer }}=11 \mathrm{~mm}-H_{\text {air }}-H_{\text {aimant }}$, the multiobjective optimization problem is then expressed as follows:

$$
\left\{\begin{array}{l}
\underset{x}{\operatorname{aax}} f=\left(f_{1}, f_{2}\right) \\
\left(\begin{array}{c}
1 \\
4.5 \\
10 \\
10 \\
2
\end{array}\right) m m \leq\left(\begin{array}{c}
H_{\text {aimant }} \\
H_{\text {air }} \\
h_{Z} \\
h_{Y} \\
e
\end{array}\right) \leq\left(\begin{array}{c}
3 \\
5.5 \\
40 \\
28 \\
10
\end{array}\right) \mathrm{mm}
\end{array}\right.
$$

Where $f_{1}(x)=\gamma^{2} / R_{0}, f_{2}(x)=\Delta z_{\gamma}$ and $x=\left[H_{\text {aimant }} H_{\text {air }} h_{Z} h_{Y} e\right]^{t}$.

To solve this problem, we used the Non-dominated Sorting Genetic Algorithm-II (NSGAII) [40]; the initial population is uniformly distributed over the variable range and counts 90 samples, the optimization process has 176 generations. 


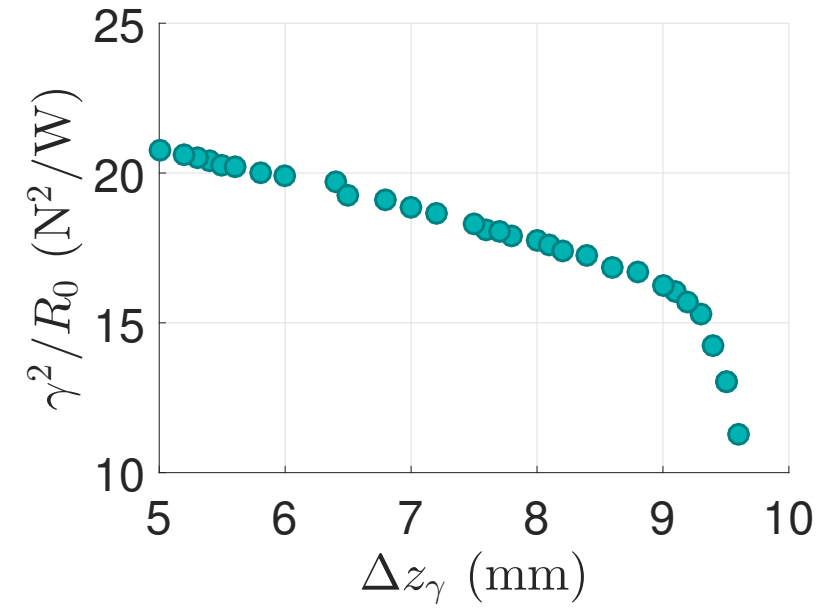

Figure C.3: Pareto-optimal solutions of the multiobjective optimization problem written in equation C.3 